\title{
Preparation of Monodisperse Hollow Core Polymer Microspheres via Two-step Dispersion Polymerization
}

\author{
Xiuting Jiang ${ }^{1,2, a}$, Shaohui Lin ${ }^{1,2, b,}$ \\ ${ }^{1}$ Green Polymer and Catalysis Technology Laboratory, \\ Soochow University; ${ }^{2}$ School of Chemical and \\ Environmental Engineering, College of Chemistry, \\ Chemical Engineering and Materials Science, Soochow \\ University. Suzhou 215123, P. R. China \\ axiuting.jiang@hotmail.com, blinshaohui@suda.edu.cn
}

\section{Garry L. Rempel}

Green Polymer and Catalysis Technology Laboratory, Soochow University

Department of Chemical Engineering, University of Waterloo, Waterloo, ON N2L 3G1, Canada grempel@uwaterloo.ca

\begin{abstract}
Monodisperse crosslinked polystyrene (PS) hollow core microspheres were successfully synthesized by two-step dispersion polymerization. The effects of the reaction parameters such as the crosslinking agent concentration, the initiator concentration and the monomer concentration on the particle sizes and size distribution were investigated. The results demonstrated that when the crosslinking agent concentration was low, the hollow microspheres had serious collapse, while the polymer particles became polydisperse with highly concentrated crosslinking agent. When increasing the initiator concentrations, particles with larger sizes and wider size distribution were obtained. If the initial monomer (styrene) weight fraction was too low, the honeycomb hollow ball or even solid particles would be formed, while if the monomer concentration was too high, the hollow microspheres appeared collapsed or ruptured.
\end{abstract}

Keywords-hollow core microspheres, dispersion polymerization, polystyrene

\section{INTRODUCTION}

Monodisperse hollow polymer microspheres with nanometer to micrometer sizes have attracted a considerable attention in recent years because of their low density, high specific surface area, surface infiltration and good permeability [1]. They can be used in many fields such as enzymes and paints [2], dyes and inks [3], drug delivery systems (DDS) [2,3], controlled storage and release, catalytic supports [4], separation and so on. Many efforts have been made by hundreds of groups worldwide to develop methods for preparing monodisperse hollow microspheres such as: self-assembly method [5], suspension polymerization [6], emulsion polymerization [7], template-assisted process [8], phase separation method [9] and so on. The template-assisted process has been developed as a facile and powerful technique for the hollow polymer microspheres synthesis. The materials of shell are

\author{
Qinmin Pan \\ Green Polymer and Catalysis Technology Laboratory, \\ Soochow University; Suzhou Key Laboratory of Green \\ Chemical Engineering; \\ School of Chemical and Environmental Engineering, \\ College of Chemistry, Chemical Engineering and Materials \\ Science, Soochow University. Suzhou 215123, P. R. China \\ qpan@suda.edu.cn (Corresponding Author)
}

flexible to choose and the size of the particle is easy to control. In the template-assisted process for hollow microspheres, the first step usually needs to form template or seeds, and the core-shell microspheres are prepared by coating shell on the surface of template or seeds. The hollow microspheres are obtained after removing the core. The size of the polymer microspheres prepared by suspension polymerization is usually between $50 \mathrm{um}$ and 2 $\mathrm{mm}$, but the distribution of resulted particles is usually wide. Emulsion polymerization is suitable for preparing monodisperse polymer spheres with diameters ranging from $100 \mathrm{~nm}$ to $200 \mathrm{~nm}$. Dispersion polymerization has been widely investigated to prepare monodisperse polymer microspheres with a size ranging from $100 \mathrm{~nm}$ to $15 \mu \mathrm{m}$. But the product is often found to have flocculation or coagulation when cross-linking agent are present. Meanwhile, the particles become oddly shaped with a broad size distribution, and the reaction is uncontrollable. In 1986, Tseng et al.[10] described experiments in which $0.3 \mathrm{wt} \%$ divinyl benzene (DVB) based on the monomer had a strong impact on the size distribution. According to the report which was published by J. S. Song [11], the post-addition procedure of DVB can regularize the size distribution of $\mathrm{P}($ St-DVB) to obtain monodisperse particles. We found that if one delayed the addition of cross-linking agents until the end of the nucleation stage, cross-linked particles of narrow size distribution could be obtained. In this paper, the DVB was added slowly after the conversion of styrene was more than $80 \%$ to get monodiseperse PS-P(St-DVB) core-shell particles. Hollow crosslinked polymer microspheres were obtained by extracting the polystyrene cores with a good solvent of polystyrene. This method was different from the conventional template-assisted process for which the template was not made in advance. In our case, the particles were in-situ produced on the first stage. So the process was simple and consecutive. 


\section{EXPERIMENTAL}

\section{A Materials}

Styrene (St) (CP grade), polyvinylpyrrolidone (PVP, K-30, Mr: 40,000) and anhydrous ethanol (AR grade) were purchased from Sinopharm Chemical Reagent Co., Ltd., China. 2,2-Azobisizobutyronitrile (AIBN) (purity $\geq 98 \%$ ) and divinyl benzene (DVB) (purity $\geq 55 \%$ ) were purchased from Aladdin Co., Ltd., China. Deionized water $\left(\mathrm{H}_{2} \mathrm{O}\right)$ was obtained from the College of Chemistry, Chemical Engineering and Materials Science of Soochow University, Suzhou, China. AIBN was recrystallized in anhydrous ethanol. St and DVB were distilled under reduced pressure in a nitrogen atmosphere to remove the inhibitor, respectively. PVP and all other reagents were used without further purification.

\section{$B$ Synthesis of monodisperse core-shell P(St-DVB) microspheres}

In this paper, we reported a novel approach to synthesize monodisperse polymer microspheres with a hollow core structure. The overall schematic illustration is shown in Scheme 1. Monodisperse PS-P(St-DVB) core-shell microspheres were prepared by two-step dispersion polymerization under the conditions listed in Table 1. The first stage was to prepare monodisperse PS-P(St-DVB) core-shell microspheres by two-step dispersion polymerization. The procedure for such polymerization was as followed: PVP were dissolved in $\mathrm{EtOH}$ and water in a four-neck glass flask reactor equipped with a mechanical stirrer. The mechanical stirring speed was kept at $200 \mathrm{rpm}$. The four-neck glass flask was purged with nitrogen for $30 \mathrm{~min}$ to remove oxygen and heated to $75^{\circ} \mathrm{C}$. Then St and AIBN were added into the reactor. The reaction mixture became milky white after 10 minutes, then the divinyl benzene (DVB) was slowly added through a constant pressure drop funnel after 1 hour. The reaction was cooled down to the room temperature after another 11 hours. After the polymerization, the resultant core-shell microspheres were purified by three cycles of ultracentrifugation, decanting and redispersion with ethanol. The polymer microspheres were dried in vacuum oven at 40 ${ }^{\circ} \mathrm{C}$ for 24 hours to get white powder.

TABLE I. RECIPE FOR THE PREPARATION OF PS-P(ST-DVB) CORE-SHELL MICROSPHERES BY TWO-STEP DISPERSION POLYMERIZATION

\begin{tabular}{cc}
\hline Ingredients & \\
\hline St & $4 \mathrm{~g}$ \\
PVP & $2 \mathrm{~g}$ \\
$\mathrm{AIBN}$ & $0.2 \mathrm{~g}$ \\
$\mathrm{EtOH}$ & $62 \mathrm{ml}$ \\
$\mathrm{H}_{2} \mathrm{O}$ & $8 \mathrm{ml}$ \\
$\mathrm{DVB}$ & $0.4 \mathrm{~g}$ \\
\hline
\end{tabular}

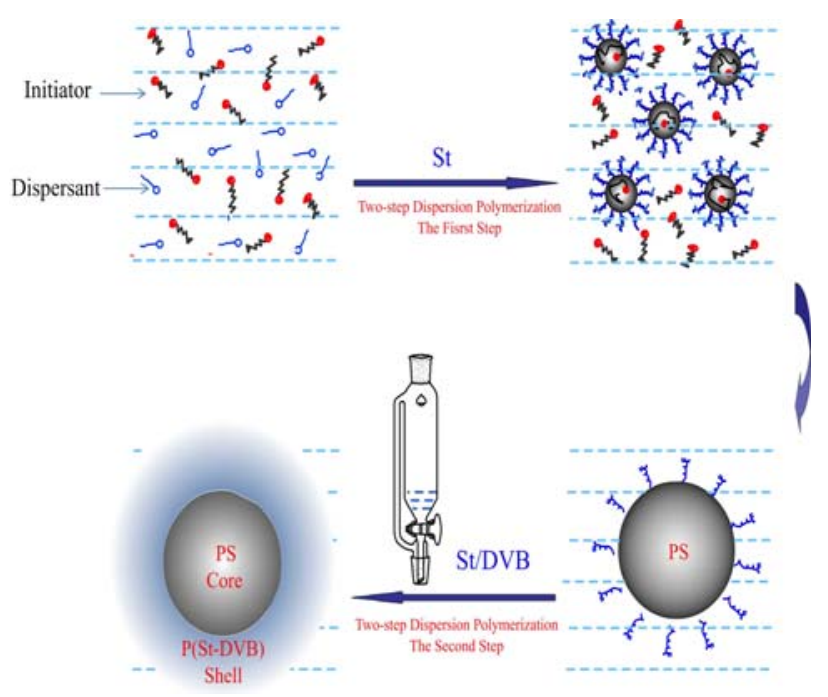

Scheme 1: Preparation of monodisperse core-shell P(St-DVB) microspheres via two-step dispersion polymerization

\section{Preparation of hollow core $P(S t-D V B)$ microspheres}

The resultant PS-P(St-DVB) core-shell microspheres were immersed in tetrahydrofuran (THF) in a single-neck glass flask reactor equipped with a condenser at $75^{\circ} \mathrm{C}$ for 24 hours. The overall schematic illustration is shown in Scheme 2. The resultant was purified by three cycles of ultracentrifugation, decanting and redispersion with ethanol. And then the polymer microspheres were dried in vacuum oven at $40^{\circ} \mathrm{C}$ for 24 hours to get white powder.

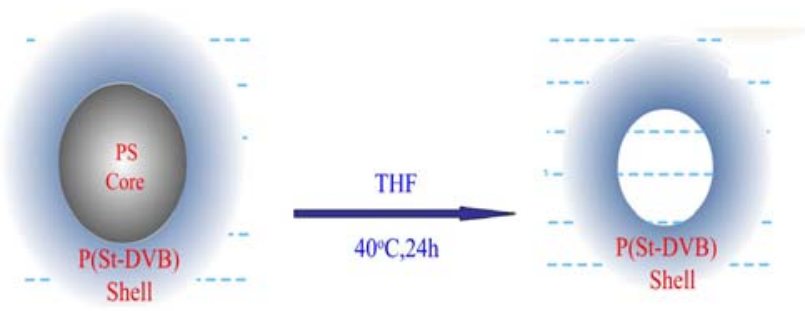

Scheme 2: Preparation of hollow core P(St-DVB) microspheres

\section{Characterization}

The Z-average size and distribution of the nanoparticles were characterized by a Malvern Zetasizer Nano ZS dynamic light scattering (DLS, UK) at $25{ }^{\circ} \mathrm{C}$. Prepared microspheres were observed by a Hitachi S-2500 C scanning electron microscope (SEM) operated at $10 \mathrm{kV}$. The microstructure and particle size were characterized by a FEI Tecnai G220 transmission electron microscopy (TEM) operated at $200 \mathrm{kV}$. 


\section{RESULTS AND DISCUSSION}

TABLE II. Z-AVERAGE AND PDI OF PS-P(ST-DVB) PARTICLES WITH DIFFERENT AMOUNT OF DVB

\begin{tabular}{ccc}
\hline $\begin{array}{c}\mathrm{DVB}(\text { weight } \\
\text { fraction relative to } \\
\text { St) }\end{array}$ & Z-Average $(\mathrm{d} / \mathrm{nm})$ & PDI \\
\hline 10 & & \\
15 & 615 & 0.020 \\
20 & 821 & 0.023 \\
25 & 950 & 0.031 \\
30 & 1210 & 0.035 \\
35 & 1405 & 0.051 \\
\hline
\end{tabular}

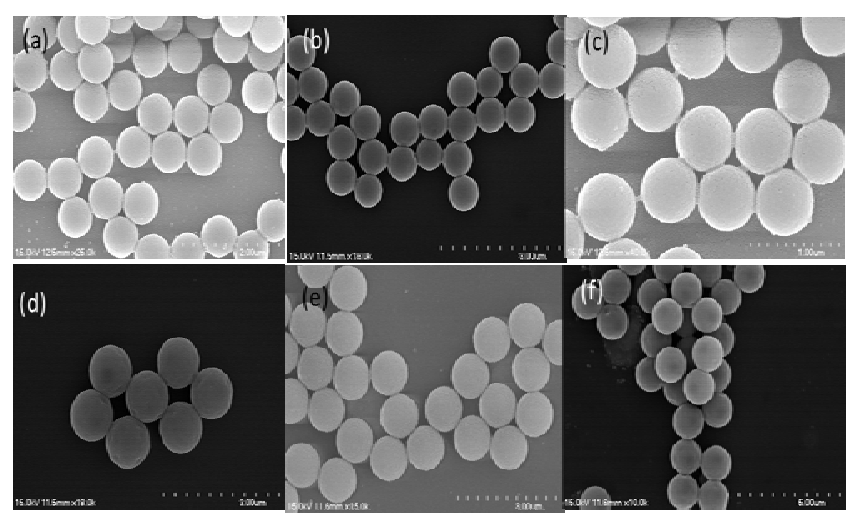

Figure 1. SEM characterizations of PS-P(St-DVB) core-shell microspheres using different amount of DVB: (a) $10 \mathrm{wt} \%$ relative to the monomer; (b) $15 \mathrm{wt} \%$ relative to the monomer; (c) $20 \mathrm{wt} \%$ relative to the monomer; (d) $25 \mathrm{wt} \%$ relative to the monomer; (e) $30 \mathrm{wt} \%$ relative to the monomer ; (f) $35 \mathrm{wt} \%$ relative to the monomer;

It has been reported that by one-stage dispersion copolymerization, with $0.35 \mathrm{wt} \%$ of DVB, the PS particles were polydisperse. With $0.67 \mathrm{wt} \%$ of DVB, the particles were no longer spherical, and with 1 wt $\%$ of DVB, the particles had irregular shapes[11]. In this paper, we prepared cross-linked polymer particles by two-step semicontinous polymerization. In this methodology, addition of the DVB was delayed until the nucleation stage was complete. Based on Table 2 and Fig. 1, when the content of DVB increased from $10 \mathrm{wt} \%$ to $30 \mathrm{wt} \%$, the average size of PS-P(St-DVB) particles would rise from $600 \mathrm{~nm}$ to $1400 \mathrm{~nm}$, and the monodisperse nanoparticles still could be prepared. In this experiment, the influence of crosslinking agent on the nucleation period could be avoided. Crosslinking agent only participated in the growth after the number of nuclei fixed, while the growth had little effect on the monodispersity. When the concentration of DVB reached $35 \mathrm{wt} \%$, the size distribution of particles became wider.

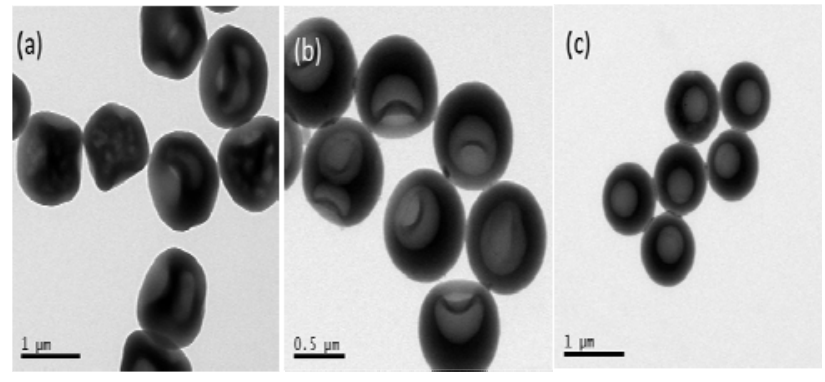

Figure 2. TEM characterizations of $\mathrm{P}(\mathrm{St}-\mathrm{DVB})$ hollow microspheres using different amount of DVB (a) $10 \mathrm{wt} \%$ relative to the monomer ; (b) $20 \mathrm{wt} \%$ relative to the monomer; (c) $30 \mathrm{wt} \%$ relative to the monomer;

The TEM images shown in Fig. 2a, b, c showed that when the DVB concentration was low, the crosslinking degree of the shell was not high, and it led to low stiffness of the hollow microspheres, which resulted in the hollow microspheres with serious collapse. The stiffness and thickness of hollow microspheres would increase with increasing the DVB concentration. When the weight concentration of DVB was $30 \mathrm{wt} \%$, the structure of hollow microspheres was neat with relatively strong shells, and the hollow microspheres were still monodisperse.

TABLE III. Z-AVERAGE AND PDI OF PS-P(ST-DVB) PARTICLES WITH DIFFERENT AMOUNT OF AIBN

\begin{tabular}{ccc}
\hline AIBN $(\mathrm{g})$ & Z-Average $(\mathrm{d} / \mathrm{nm})$ & PDI \\
\hline 0.20 & 815 & 0.037 \\
0.25 & 836 & 0.039 \\
0.30 & 978 & 0.045 \\
0.35 & 1352 & 0.090 \\
0.40 & 1796 & 0.105 \\
\hline
\end{tabular}
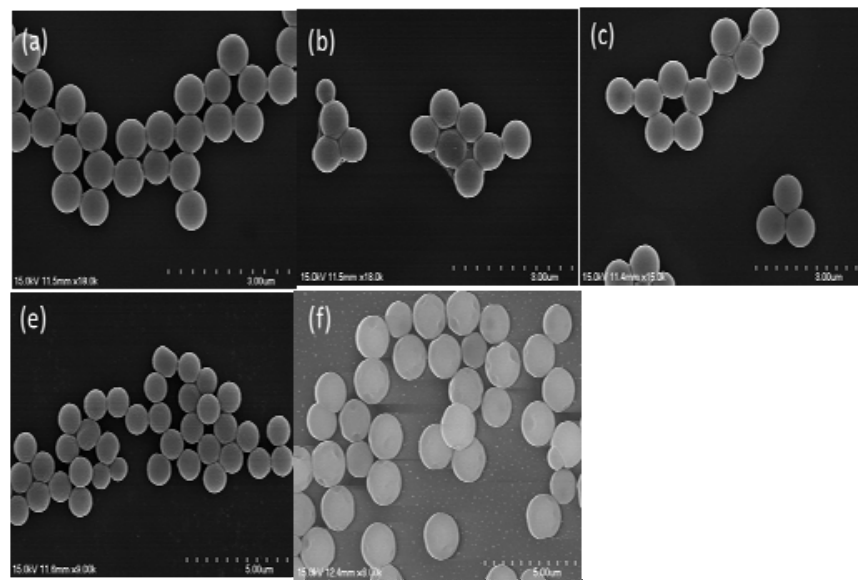

Figure 3. SEM characterizations of PS-P(St-DVB) core-shell microspheres with different amount of AIBN: (a) $0.20 \mathrm{~g}$; (b) $0.25 \mathrm{~g}$; (c) $0.30 \mathrm{~g}$; (d) $0.35 \mathrm{~g}$; (e) $0.40 \mathrm{~g}$; 
The average sizes and size distribution of hollow core polymer microspheres prepared with different initiator concentrations are given in Table 3, and the optical microscope photographs are present in Fig. 3. As seen in Table 3 and Fig. 3, we could find that increasing initiator concentration led to bigger average sizes and wider size distribution. When the dosage of initiator increased to a certain extent, the microsphere surface roughness increased significantly. Especially when the initiator content increased to $0.4 \mathrm{~g}$, the PDI of polymer microspheres was 0.105 , and the monodispersity of polymer microspheres deteriorated rapidly.

In the two-step dispersion polymerization, before there was crosslinking agent, when increasing initiator dosage, the number of initially generated radicals increased, and the polymerization rate of styrene was enhanced. With a fixed amount of stabilizer, more polymer nuclei led to the stabilizer deficiency, and then the stabilizer could not stabilize the nuclei in time, which reduced the stability of the particles. As a result, larger size and wider size distribution particles were obtained when increasing the AIBN concentration.

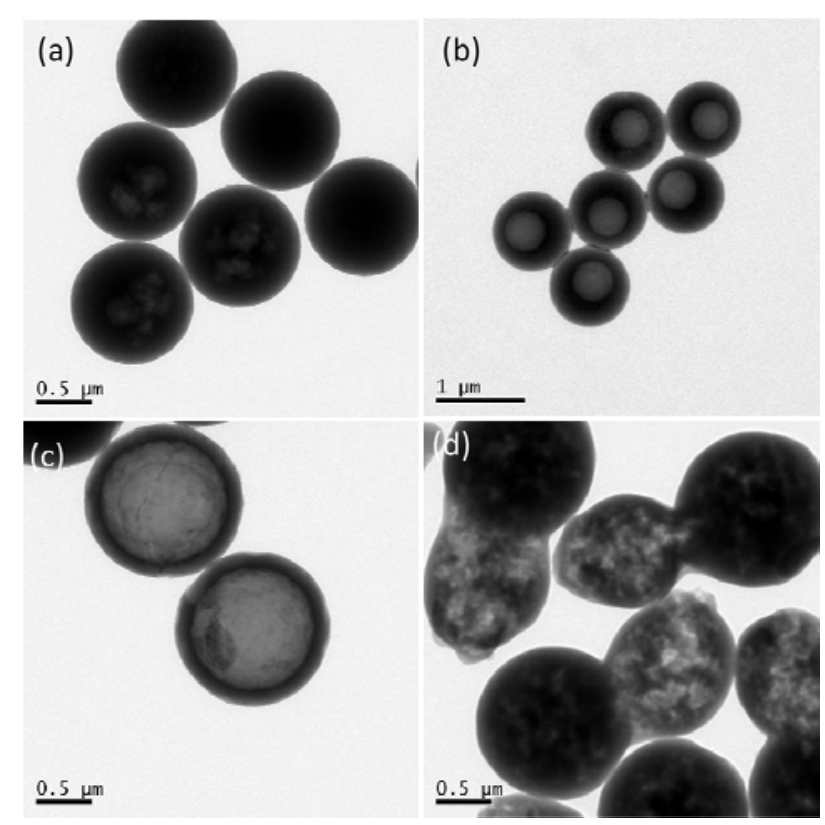

Figure 4. TEM images of hollow particles with different concentrations of monomer (a) $4.1 \mathrm{vol} \%$, (b) $6.3 \mathrm{vol} \%$, (c) $8.5 \mathrm{vol} \%$, (d) $10.7 \mathrm{vol} \%$

It has been reported that with monomer concentration increasing, the polymer particles sizes increased[12]. When the St concentration were higher than $15 \mathrm{vol} \%$ or lower than 2.5 vol $\%$, polydisperse particles were obtained[13]. TEM images of hollow particles with different concentrations of monomer are shown in Fig. 4.

In this set of experiments, the monomer concentration was varied from $4.1 \mathrm{vol} \%$ to $10.7 \mathrm{vol} \%$ and the other components were kept constant. Fig. 4 showed that microspheres were almost solid when the monomer volume fraction was low. The microspheres collapsed when the monomer volume fraction was too high. Only when the monomer volume fraction was moderate, especially from $6.3 \mathrm{vol} \%$ to $8.5 \mathrm{vol} \%$, the neat hollow microspheres could be obtained.

In the two-step dispersion polymerization, when the crosslinking agent was added, most of the monomer had been converted into polymer, and polymerization reaction rate had been falling rapidly. For different starting monomer concentrations, there was no much difference on the monomer conversion rate. If the initial monomer concentration was high, the residual monomer concentration was still high when the crosslinking agent was added, then these monomers could be copolymerized with DVB, so the crosslinking layer would be formed by settling to the particle surface. The crosslinking degree of shell would reduce if more St and DVB copolymerized, and as a result, the rigidity of hollow crosslinking microspheres would be reduced dramatically, and the hollow microspheres would rupture, which is shown in Fig. 4 (d). On the other hand, if the initial monomer weight fraction was low, residual monomer became very little when the crosslinking agent was added. So the surface of hollow microspheres was mainly formed by the DVB polymerization, and the crosslinking density was high. The rigidity of hollow crosslinking microspheres was improved, and the structure of the hollow ball was complete, as shown in Fig. 4 (b), (c). However, if the initial monomer volume fraction was too low, crosslinking layer structure might be too dense, and the kernel polymer couldn't be dissolved, in which the honeycomb or even solid ball would be formed, as shown in Fig. 4 (a).

\section{CONCLUSIONS}

Hollow crosslinked polystyrene microspheres with different shell thicknesses and crosslinking degree were prepared by a facile route, in which a two-step disperse polymerization and subsequent removal of non-crosslinked polystyrene core with THF were included. The feed of AIBN, monomer and the crosslinking degree had much effect on the core-shell microspheres morphology and the shell thickness. The hollow core microspheres with narrow size distribution, different sizes and shell thickness were synthesized and confirmed further by DLS, SEM and TEM characterization.

\section{ACKNOWLEDGEMENTS}

Financial support from the National Nature Science Foundation of China (No. 21176163), Suzhou Industrial Park, Shandong Yuhuang Chemical Co. Ltd., the Priority Academic Program Development of Jiangsu Higher Education Institutions, the Program of Innovative Research Team of Soochow University, and Natural Science and Engineering Council of Canada are gratefully acknowledged. 


\section{REFERENCES}

[1] Dong A. G., Wang Y. J., Wang D. J., Fabrication of hollow zeolite microcapsules with tailored shapes and functionalized interiors . Microp Mesop Mater , 64 : 69-81, 2003.

[2] Gill I., Ballesteros A., Encapsulation of biologicals within silicate, siloxane, and hybrid sol-gel polymers: an efficient and generic approach. J. Am. Chem. Soc. 120, 8587-8598, 1998.

[3] Wilcox, D. L., Sr., Berg, M., Bernat, T., Kellerman, D., Cochran, J. K., Jr., Eds., Hollow and solid spheres and microspheres: science and technology associated with their fabrication and application. Materials Research Society Proceedings: Pittsburgh, Vol. 372, 1995.

[4] Morris C. A., Anderson M. L., Stroud R. M., Merzbacher C. I., Rolison D. R., Silica sol as a nanoglue: flexible synthesis of composite aerogels, Science, 284, 622-624, 1999.

[5] Zhang P, Feng NP, Wu P Y, Polymeric hollow nanospheres and the application in pharmacy there of $[\mathrm{J}]$. Progress in Pharmaceutical Sciences, 30 (8) : 350-353 . (in Chinese), 2006.

[6] Kim B S , Kim J W, Suh K D, Poly (methyl methacrylate) multihollow particles by water in oil in water emulsion polymerization [J]. J. Appl. Polym. Sci. 76 : 38-44, 2000.

[7] Okubo M, Konishi Y, Inohara T, Production of hollow polymer particles by suspension polymerizations for ethylene glycoldimethacrylate/toluene droplets dissolving styrene-methylmethacrylate copolymers [J]. J. Appl. Polym. Sci. 86 (5):1087-1091, 2002.

[8] Minami H, Kobayashi H, Okubo M., Preparation of hollow polymer particles with a single hole in the shell by SASEP [J] . Langmuir, 21 : 5655-5658, 2005.

[9] Chang M W, Stride E, Edirisinghe M., A new method for the preparation of monoporous hollow microspheres [J]. Langmuir, 26 : 5115-5121, 2010.

[10] Tseng C. M., Lu, Y. Y., El-Aasser, M. S., Vanderhoff, J. W., Uniform polymer particles by dispersion polymerization in alcohol. J. Polym. Sci., Part A: Polym. Chem. 24, 2995, 1986.

[11] J. S. Song, Mitchell A. Winnik, Cross-linked, monodisperse, micron-sized polystyrene particles by two-stage dispersion polymerization, macromolecules. 38, 8300-8307, 2005.

[12] Fen Zhang, Le Cao, Wantai Yang, Preparation of monodisperse and anion-charged polystyrene microspheres stabilized with polymerizable sodium styrene sulfonate by dispersion polymerization, Macromol. Chem. Phys. 211, 744-751, 2010.

[13] Hongtao Zhang, Jinxia Huang, Bingbing Jiang, Kinetics of polymerization and particle stabilization mechanism on dispersion copolymerization of styrene and divinylbenzene, J. Appl. Polym. Sci. Vol. 85, 2230-2238, 2002. 